\title{
Neuroprotective Effect of Arctigenin via Upregulation of P-CREB in Mouse Primary Neurons and Human SH-SY5Y Neuroblastoma Cells
}

\author{
Nan Zhang ${ }^{1, \dagger}$, Qingping Wen ${ }^{2, \dagger}$, Lu Ren ${ }^{3}$, Wenbo Liang ${ }^{4}$, Yang Xia ${ }^{5}$, Xiaodan Zhang ${ }^{1}$, \\ Dan Zhao ${ }^{1}$, Dong Sun ${ }^{1}$, Yv Hu ${ }^{1}$, Haiguang Hao ${ }^{1}$, Yaping Yan ${ }^{6}$, Guangxian Zhang ${ }^{6}$, \\ Jingxian Yang ${ }^{1, *}$ and Tingguo Kang ${ }^{1, *}$
}

1 School of Pharmacy, Liaoning University of Traditional Chinese Medicine, Dalian 116600, Liaoning, China; E-Mails: nanzhang2002@hotmail.com (N.Z.); zxd20060205009@aliyun.com (X.Z.); danzhao88@outlook.com (D.Z.); s43792211@sina.com (D.S.); cynthiafishu@aliyun.com (Y.H.); haiguang_hao@aliyun.com (H.H.)

2 First Affiliated Hospital, Dalian Medical University, Dalian 116011, Liaoning, China; E-Mail: wqp.89@163.com

3 Liaoning University of Traditional Chinese Medicine, Shenyang 110847, Liaoning, China; E-Mail: renlu2008.student@sina.com

4 Medical College of Dalian University, Dalian 116600, Liaoning, China; E-Mail: dllwb@126.com

5 College of Engineering, University of California Berkeley, Berkeley, CA 94720, USA;

E-Mail: xiayang9999@vip.sina.com

6 Department of Neurology, Thomas Jefferson University, Philadelphia, PA 19107, USA; E-Mails: yaping.yan@snnu.edu.cn (Y.Y.); guang-xian.zhang@jefferson.edu (G.Z.)

$\dagger$ These authors contributed equally to this work.

* Authors to whom correspondence should be addressed;

E-Mails: jingxianyang630520@gmail.com (J.Y.); tingguokang@hotmail.com (T.K.);

Tel.: +86-411-8758-6009 (J.Y.); +86-411-8758-6005 (T.K.);

Tel./Fax: +86-411-8758-6078 (T.K.).

Received: 10 May 2013; in revised form: 22 July 2013 / Accepted: 23 August 2013 /

Published: 10 September 2013

Abstract: Arctigenin (Arc) has been shown to act on scopolamine-induced memory deficit mice and to provide a neuroprotective effect on cultured cortical neurons from glutamate-induced neurodegeneration through mechanisms not completely defined. Here, we investigated the neuroprotective effect of Arc on H89-induced cell damage and its 
potential mechanisms in mouse cortical neurons and human SH-SY5Y neuroblastoma cells. We found that Arc prevented cell viability loss induced by H89 in human SH-SY5Y cells. Moreover, Arc reduced intracellular beta amyloid (A $\beta$ ) production induced by H89 in neurons and human SH-SY5Y cells, and Arc also inhibited the presenilin 1(PS1) protein level in neurons. In addition, neural apoptosis in both types of cells, inhibition of neurite outgrowth in human SH-SY5Y cells and reduction of synaptic marker synaptophysin (SYN) expression in neurons were also observed after H89 exposure. All these effects induced by $\mathrm{H} 89$ were markedly reversed by Arc treatment. Arc also significantly attenuated downregulation of the phosphorylation of CREB (p-CREB) induced by H89, which may contribute to the neuroprotective effects of Arc. These results demonstrated that Arc exerted the ability to protect neurons and SH-SY5Y cells against H89-induced cell injury via upregulation of $\mathrm{p}$-CREB.

Keywords: arctigenin; neuroprotection; beta amyloid (A $\beta)$; p-CREB

\section{Introduction}

Arctigenin (Arc) is the main active constituent that is extracted and isolated from the fruit of Arctium lappa L., which has been used as an herbal medicine for its antipyretic and anti-inflammatory actions. Arc itself has antioxidant, anti-inflammatory, anti-tumor and immunomodulatory effects [1-3]. Moreover, Arc exerts a neuroprotective effect on both glutamate-induced neurotoxicity in primary neurons and scopolamine-induced learning and memory deficits in mice with Alzheimer's disease (AD) $[4,5]$. However, the underlying mechanism remains elusive.

Beta-amyloid (A $\beta$ ) peptide is the central player in the amyloid cascade hypothesis that describes AD etiology [6]. Cyclic AMP response element-binding protein (CREB) is a nuclear transcription factor that is activated by phosphorylation at serine 133 [7]. Activation of CREB is essential for the formation and retention of memory, which is the main physiological parameter of Alzheimer's disease [8]. The process of CREB activation is also considered to be a major mechanism in the promotion of neuronal growth and survival [9]. Reduced phosphorylation of CREB (p-CREB), which is considered to be one of the consequences of $A \beta$-induced neurotoxicity, has been observed in the postmortem brains of $\mathrm{AD}$ patients, $\mathrm{A} \beta$-treated neurons [10] and in Tg-AD mice that overexpress $\mathrm{A} \beta$ [11]. CREB can be activated by multiple signaling pathways, including protein kinase A (PKA). H89, a selective pharmacological PKA inhibitor, possesses the ability to suppress CREB phosphorylation in a concentration-dependent manner [12] and, thus, has been widely used as a laboratory agent in inhibition of CREB phosphorylation [13-17]. Recent studies have reported that H89 reduced the viability of primary cortical neurons [18]. In the present study, we thus used H89 to induce neural cell damage via downregulation of p-CREB [19,20] and investigated if Arc protects neural cells against CREB inactivation. 


\section{Results and Discussion}

\subsection{Arc Protects Human SH-SY5Y Cells from H89-Induced Reduction of Cell Viability}

We first did a dose-response study of H89 on human SH-SY5Y cells. Cells were incubated with various concentrations of H89 $(1-400 \mu \mathrm{M})$ for $1 \mathrm{~h}$. Cell survival was assessed by MTT (3-(4,5-dimethyl-2-thiazolyl)-2,5-diphenyl-2-tetrazolium bromide) assay. H89 (10-400 $\mu \mathrm{M})$ resulted in $28 \%-82 \%$ cell death (Figure 1A). Furthermore, H89 has been reported to induce downregulation of p-CREB at $50 \mu \mathrm{M}$ [20]. We therefore used this dose in the next experiments.

To investigate whether Arc could rescue the loss of cell viability, human SH-SY5Y cells were treated with H89 $(50 \mu \mathrm{M}, 1 \mathrm{~h})$ before being incubated with Arc $(0.25-10 \mu \mathrm{M}$ for $24 \mathrm{~h})$. Cell viability was assessed by MTT assay. Results showed that the viability of H89-treated cells was reduced to $61.90 \% \pm 9.79 \%$ in comparison with the control group $(100 \%, p<0.05$, Figure $1 \mathrm{~B})$; Arc significantly increased the viability, with a maximal effect at $0.5 \mu \mathrm{M}(99.86 \% \pm 1.75 \%)(v s . \mathrm{H} 89$-control, $p<0.05$, Figure 1B). Arc decreased cell viability at concentrations higher than $1 \mu \mathrm{M}$, indicated that Arc exerted a neuroprotective effect at a proper range of concentrations $(0.25-0.5 \mu \mathrm{M})$ against H89-induced cell damage.

Figure 1. Protective effects of arctigenin (Arc) on human SH-SY5Y cells from H89-induced cell injury. (A) Human SH-SY5Y cells viability treated with different concentrations of $\mathrm{H} 89$ for $1 \mathrm{~h}$; (B) Viability of cells treated with different concentrations of Arc after being exposed to $\mathrm{H} 89(50 \mu \mathrm{M})$ for $1 \mathrm{~h}$. Cell viability was detected by MTT assay. Values represent the mean $\pm \mathrm{SD}$ from three separate experiments $(n=9)$. ${ }^{*} p<0.05$; $* * p<0.01 ; * * * p<0.001$ vs. the control; \# $p<0.05$ vs. the H89 group.
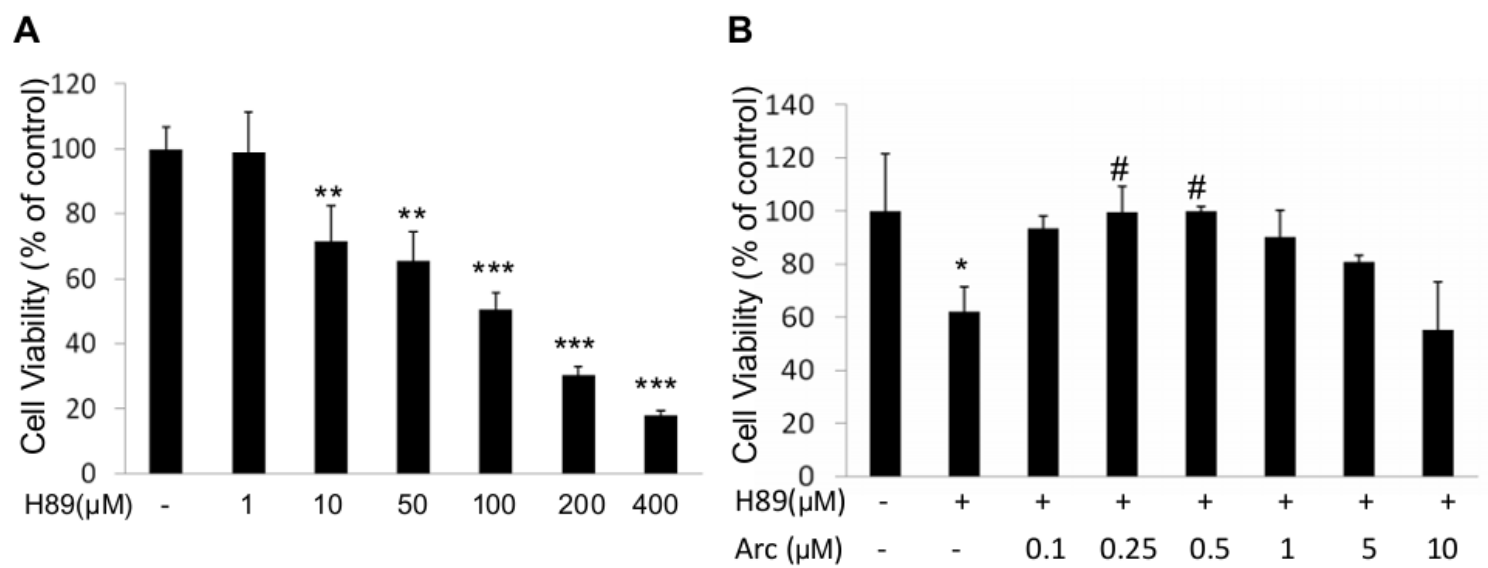

\subsection{Arc Attenuates Aß Production Induced by H89}

Mouse cortical neurons were cultured and identified with immunostaining of neural marker, Neurofilament M (NF-M) (Figure 2A). A $\beta(35-42)$ expression in neurons and SH-SY5Y cells was determined by immunostaining with anti-A $\beta(35-42)$ antibody. As shown in Figure $2 \mathrm{~B}$, the immunoreactivity of $A \beta$ in H89-treated groups was greater than control groups, whereas it was significantly decreased after treatment with Arc for $24 \mathrm{~h}$. Quantitative analysis of the immunocytochemistry suggested that the levels of $\mathrm{A} \beta$ in H89-treated neurons and SH-SY5Y cells were $156.93 \% \pm 4.65 \%$ and $116.74 \% \pm 1.61 \%$ of the control group, respectively (Figure $2 \mathrm{C}, p<0.01$, 
$p<0.01$, vs. control 100\%). Arc treatment attenuated the H89-induced increase of A $\beta$ to $115.42 \% \pm 3.29 \%$ and $110.70 \% \pm 3.28 \%$, respectively (Figure $2 \mathrm{C}, p<0.01, p>0.05$, vs. H89 group).

We further investigated BACE1 and PS1 production by RT-PCR and ELISA. The results indicated that the mRNA expression of BACE1 and PS1 was not affected by H89 and H89 plus Arc treatment (Figure 2D,E) and with a similar protein level pattern of BACE1 (Figure 2F). However, the protein level of PS1 assayed by ELISA was significantly increased by $\mathrm{H} 89$ exposure, which was significantly reversed by the treatment with Arc (Figure 2F, 126.24\% $\pm 2.94 \%$ of the H89 group vs. control, 100\%, $p<0.01 ; 96.98 \% \pm 6.44 \%$ of the H89 + Arc group $v s .126 .24 \% \pm 2.94 \%$ of the H89 group, $p<0.01$ ). These data indicated that the effect of neurons incubated with Arc decreased the A $\beta$ level induced by H89, which was not associated with significant changes in mRNA and the protein levels of BACE1. By contrast, the effect of $A \beta$ inhibition was associated with a reduction of the PS1 protein level, indicating that the effect of Arc on the reduction of intracellular $A \beta$ may be associated with the reduction of the PS1 protein.

Figure 2. Arc attenuated $A \beta 35-42$ production induced by $\mathrm{H} 89$ by reducing the PS1 protein level. (A) Neurons identified by immunostaining of neural marker, NF-M (green), and 4',6-diamidino-2-phenylindole (DAPI, blue). Scale bar $=25 \mu \mathrm{m}$; (B) Neurons and SH-SY5Y cells were immunostained using anti-A $335-42$ antibody (red) and DAPI (blue). Scale bar $=20 \mu \mathrm{m}$; (C) Average fluorescence intensity of $\mathrm{A} \beta$ immunostaining was measured in triple cultures; (D) mRNA of BACE1 and PS1 were analyzed by RT-PCR; (E) Quantitative analysis of the relative mRNA levels of BACE1 and PS1 by Image J software; (F) Cell supernatants of neurons were assayed using ELISA. The data were expressed as the mean $\pm \mathrm{SD}(n=6) ; * * p<0.01 v s$. the control; \#\# $p<0.01 v s$. the H89 group.

A

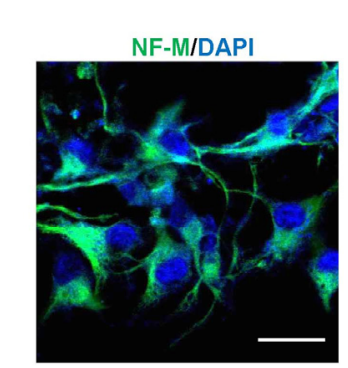

B

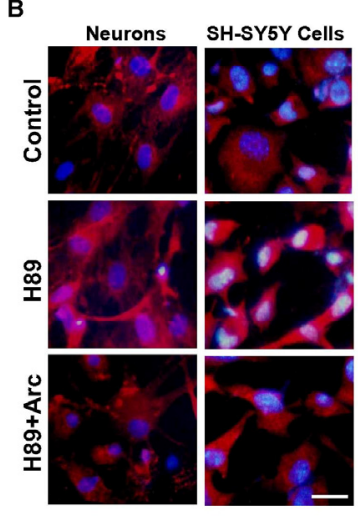

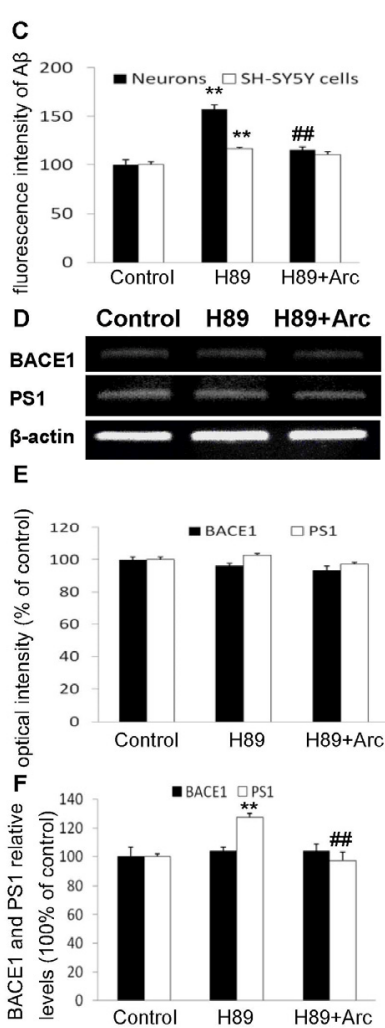




\subsection{Arc Protects Neuron-Like Cells and Neurons against Apoptosis Induced by H89}

To evaluate whether Arc protected neural cells form apoptosis, Hoechst33258 staining was performed, both in cortical neurons and SH-SY5Y cells (Figure 3A). The cells were incubated with $\mathrm{H} 89(50 \mu \mathrm{M})$ for $1 \mathrm{~h}$ prior to exposure to $\operatorname{Arc}(0.5 \mu \mathrm{M})$ for $24 \mathrm{~h}$. The control groups showed intact and relatively large nuclei, whereas H89-treated cells showed an increase in condensed nuclei. Arc treatment reduced the number of condensed nuclei significantly compared with the H89 groups. Quantitative analysis showed that the apoptotic rate was $31.67 \% \pm 2.08 \%$ in SH-SY5Y cells treated with H89 ( $p<0.001$, compared with the control, Figure 3B) and $17.07 \% \pm 1.25 \%$, when cells were cultured with Arc ( $p<0.001$, compared with the H89 group). Meanwhile, the percentage of apoptotic neurons was $26.79 \% \pm 1.49 \%$ in neurons in the H89 group ( $p<0.001$, compared with the control) and $13.33 \% \pm 1.53 \%$, when neurons were incubated with Arc ( $p<0.001$, compared with the H89 group). All these results indicated that Arc effectively protected neuron-like cells and cortical neurons against H89-induced apoptosis.

Figure 3. Arc inhibited apoptosis induced by H89 and restored neurite outgrowth and synaptic markers expression against H89-induced disorders. (A) Hoechst33258 staining was performed in mouse cortical neurons and SH-SY5Y cells. Scale bar $=25 \mu \mathrm{m}$; (B) Quantitative analysis of cell apoptosis; (C) Morphological characteristic of SH-SY5Y cells were observed using phase-contrast microscopy. Scale bar $=25 \mu \mathrm{m}$; (D) In 10 random fields, the number of neurites was counted, and the ratio of neurites to cell bodies was calculated; (E) Neurons were immunostained with synaptophysin (SYN, red) and DAPI (blue). Scale bar $=20 \mu \mathrm{m}$; (F) Average fluorescence intensity of SYN immunostaining was assessed in 10 random areas. All the data were expressed as the mean $\pm \operatorname{SD}(n=9)$; $* * * p<0.001$ vs. the control; \# $p<0.05$; \#\# $p<0.01$; \#\#\# $p<0.001$ vs. the H89 group.

A
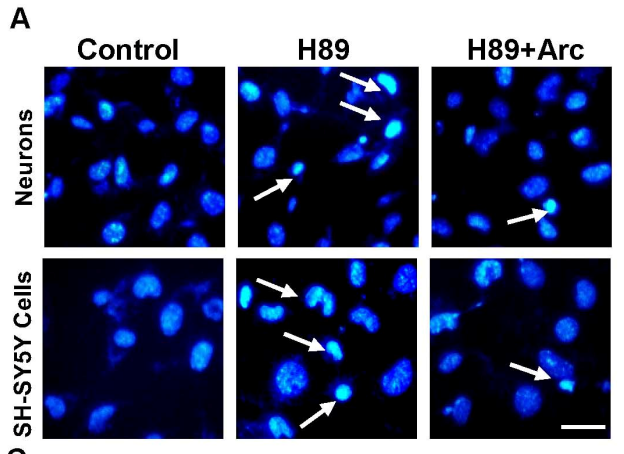

C Control

H89
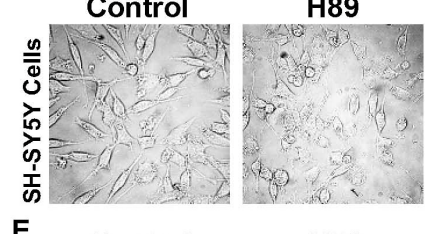

H89+Arc

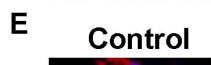

H89
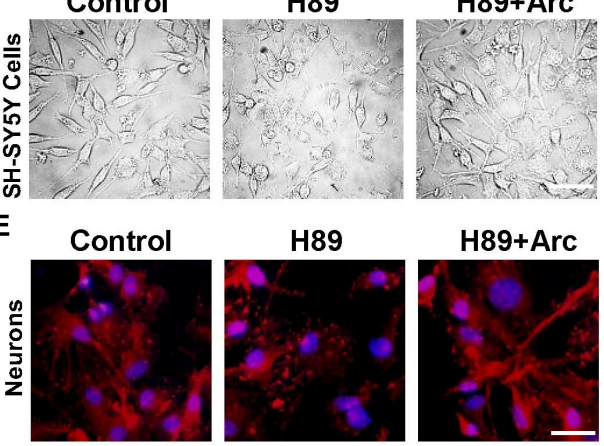

H89+Arc

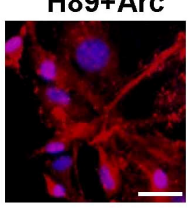

B
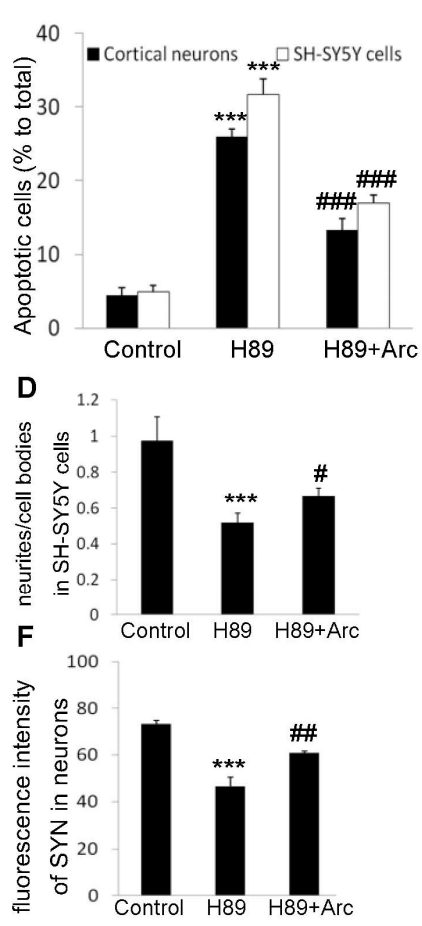


\subsection{Arc Restores Neurite Outgrowth and SYN Expression against H89-Induced Disorders}

To assess the neuroprotection of Arc on synaptic impairment induced by H89, We first examined the neurite outgrowth in human SH-SY5Y cells. Cells were incubated with Arc $(0.5 \mu \mathrm{M}, 24 \mathrm{~h})$ after being treated with $\mathrm{H} 89$ for $1 \mathrm{~h}$. For quantification of neurite outgrowth, cell morphology was observed using phase-contrast microscopy. The number of neurites was counted, and the ratio of neurites to cell bodies was calculated. H89-treated cells displayed polygonal cell bodies and short processes. Cells incubated with Arc significantly reversed the appearance of neurite short processes induced by H89 (Figure 3C). As shown in Figure 3D, the ratio of neurites to cell bodies in H89-treated cells significantly decreased by $51.80 \% \pm 5.48 \%$ of the control group ( $p<0.001$ ), while Arc-treated cells exhibited a higher value compared to the H89-treated cells $(64.78 \% \pm 1.31 \%$ vs. $51.80 \% \pm 5.48 \%, p<0.05)$.

In neurons, we performed an analysis of immunocytochemical staining of SYN, which is an integral membrane glycoprotein of synaptic vesicles, which has been widely used as a synaptic marker to investigate synaptic reinnervation and synaptogenesis [21]. The immunostaining of SYN was markedly decreased in the H89-treated neurons (Figure 3E,F, $p<0.001$, compared with the control), indicating the occurrence of synaptic degeneration. However, the decrease of SYN induced by H89 was greatly alleviated after Arc treatment (Figure 3E,F, $p<0.01$ ). Moreover, we treated neurons and SH-SY5Y cells with Arc in the absence of H89, and we found that there was no difference between the control groups and Arc $(0.5 \mu \mathrm{M})$ alone (data not shown). These findings suggested that Arc protected neural cells against H89-induced synaptic impairment.

\subsection{CREB May Be Involved in the Neuroprotection of Arc against H89-Induced Cell Injury}

We then studied the correlation between CREB phosphorylation and the neuroprotective potential of Arc in neural cells. The expression of CREB mRNA was first assessed following amplification of RNA isolated from neurons. There was no significant change in the mRNA level of CREB in neurons-treated with $\mathrm{H} 89$ compared with the control, while cells incubated with Arc showed an increase (not significant) in the level of CREB mRNA (Figure 4A,B). Next, immunocytochemistry was performed to visualize the activation of CREB by Arc in neurons and human SH-SY5Y cells. H89-treated cells expressed most of the p-CREB in their cytoplasm, and the addition of Arc $(0.5 \mu \mathrm{M})$ resulted in the translocation of p-CREB immunoreactivity into the nuclear region (Figure 4C). As shown in Figure 4D, treatment with $\mathrm{H} 89$ led to a decrease of the density of immunoreactive p-CREB in both cortical neurons $(62.30 \% \pm 4.06 \%$, compared with the control, $p<0.001)$ and SH-SY5Y cells $(65.46 \% \pm 6.46 \%$, compared with the control, $p<0.01)$. Whereas the effects were significantly alleviated by treatment with Arc in neurons $(80.57 \% \pm 3.34 \%$, compared with the H89 group, $p<0.05)$ and SH-SY5Y cells $(82.04 \% \pm 1.98 \%$, compared with the H89 group, $p<0.05)$. These data suggested that Arc may upregulate CREB, which contributes to its neuroprotective activity. 
Figure 4. Arc attenuated the inhibition of the phosphorylation of cyclic AMP response element-binding protein (CREB) (p-CREB) induced by H89 in SH-SY5Y cells and neurons. (A) The mRNA of CREB was analyzed by RT-PCR in neurons; (B) The relative optical density of CREB mRNA was acquired by Image $J(N I H$ Image $J 1.38 \times$, National Institutes of Health, Bethesda, MD, USA); (C) Neurons and SH-SY5Y cells were immunostained for p-CREB (red) and DAPI (blue). Scale bar $=20 \mu \mathrm{m}$; (D) The average fluorescence intensity of p-CREB immunostaining was assessed in 10 random areas. All the data were expressed as the mean $\pm \mathrm{SD}(n=3) ; * * p<0.01 ; * * * p<0.001 v s$. the control; \# $p<0.05$ vs. the H89 group.

A

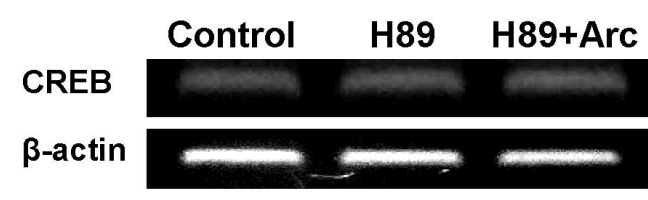

C Control
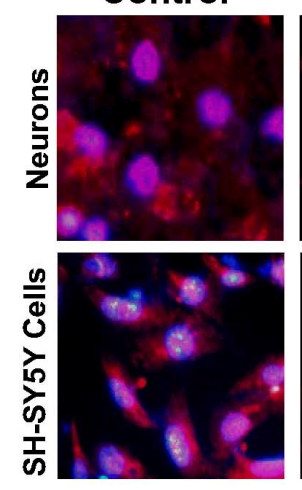

H89
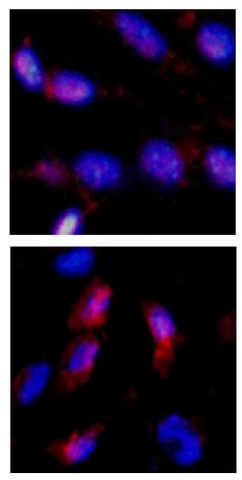

H89+Arc

\section{B}

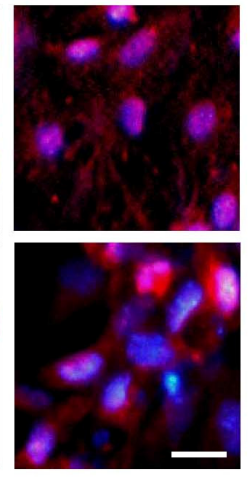

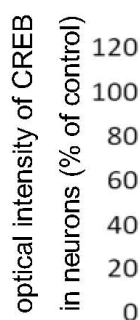

D
Control H89 H89+ArC

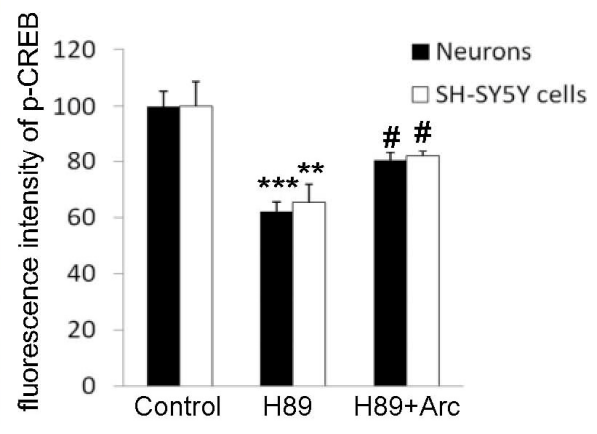

\subsection{Discussion}

In this study, we demonstrated for the first time that Arc attenuation of H89-induced enhancement of $\mathrm{A} \beta$ production in primary neurons and SH-SY5Y cells reduced PS1 protein expression in neurons. Moreover, Arc curtailed H89-induced cell viability inhibition, neural apoptosis and synaptic impairment, which may be associated with the reduction of H89-induced CREB inactivation, and exerted neuroprotective effects.

The presence of $\mathrm{A} \beta$ has been implicated as one of the most important pathogenic traits of AD. Previous studies have demonstrated that $\mathrm{A} \beta$ induces an inflammatory response, oxidative stress, mitochondrial dysfunction and neural apoptosis, resulting in neurodegeneration [22,23]. Therefore, reducing the level of $A \beta$ in neurons could have profound effects on AD pathophysiology. Here, we found that exposure of SH-SY5Y cells and primary neurons to H89 elicited a significant increase in intracellular $A \beta$. The H89-induced increase in $A \beta$ was attenuated by treatment with Arc, showing that Arc can block this effect of $\mathrm{H} 89$ on $\mathrm{A} \beta$ levels.

$\mathrm{A} \beta$ is derived from the cleavage of amyloid precursor protein (APP). APP undergoes ectodomain shedding at two alternative sites; one is mediated by BACE1 and results in the formation of 
membrane-associated $C$-terminal fragments (CTFs), termed C99s. The subsequent $\mathrm{PS} / \gamma$-secretase-mediated cleavage of $\mathrm{C} 99$ at the $\gamma$-site releases A $\beta$ peptides of different lengths [24]. The ability of Arc to depress the H89-induced increase in A $\beta$ suggests that Arc may play a role in $A \beta$ production during APP processing. Consistent with this hypothesis, we observed that while the mRNA levels of BACE1 and PS1 were not altered in mouse primary neurons exposed to H89 or H89 plus Arc, the PS1 protein level was significantly increased by H89 and reversed by Arc treatment, suggesting that Arc reduced A $\beta$ production by decreasing the PS1 protein level. It should be noted that the reduction in A $\beta$ levels by Arc was only associated with the PS1 protein, and not mRNA, levels. Thus, how Arc reduces PS1 protein expression warrants further investigation.

In the present study, we also found that Arc treatment could inhibit H89-induced depression of cell viability and neural apoptosis in both types of cells (cortical neurons and SH-SY5Y cells), thereby rescuing them from neurodegeneration and confirming the neuroprotective action of Arc against H89. It is known that synaptic impairment and synapse loss are events in the early stages of AD [25]. Therefore, restoring synaptic integration in neurons could be beneficial for the treatment of AD. Previous studies have shown that H89 robustly inhibits neurite formation in PC12 cells [26], and another PKA inhibitor is shown to dramatically suppress the levels of SYN in neurons [27]. Here, we found that neurite outgrowth was significantly inhibited by H89 in SH-SY5Y cells and that the expression of SYN was decreased by H89 in cortical neurons. Moreover, administration of Arc to the two types of cells studied resulted in a significant alleviation of the H89-induced reduction in neurite outgrowth in SH-SY5Y cells and of the H89-induced disturbance of SYN expression, further demonstrating the neuroprotective effect of Arc.

In addition to its roles in other physiological processes, including Long-term Potentiation (LTP), CREB can be activated by PKA [28] to regulate the transcription of an array of pro-survival and anti-apoptotic genes [29]. Thus, it is not surprising that the consequences of disrupting CREB function are severe. H89 is a selective PKA inhibitor that has been reported to significantly inhibit the activation of CREB [12]. In this study, we first examined the mRNA level of CREB and found that H89 and Arc did not alter the mRNA expression. We further performed immunocytochemistry to visualize p-CREB; we found that exposure to H89 elicited a significant inhibition of CREB phosphorylation in both cortical neurons and SH-SY5Y cells. Administration of Arc reversed the H89-induced downregulation of p-CREB in these cells. However, further experiments, such as Western blot, should perform to attain the ratio of pCREB/CREB to confirm the results. Collectively, these results indicate that restoration of CREB phosphorylation by Arc in H89-treated cells accounts for the neuroprotective effects of Arc.

\section{Experimental Section}

\subsection{Cell Culture}

Primary cortical neurons were prepared from neonatal (P0-P2) mouse brain using methods described previously [30]. A neuron-like cell line, human SH-SY5Y cells, was plated at a density of $1.0 \times 10^{5} / \mathrm{mL}$. The cultures were grown in Dulbecco's modified Eagle's medium (DMEM) 
supplemented with $10 \%$ fetal bovine serum (FBS), $100 \mathrm{U} / \mathrm{mL}$ penicillin and $100 \mu \mathrm{g} / \mathrm{mL}$ streptomycin (all from Gibco, New York, NY, USA) at $37{ }^{\circ} \mathrm{C}$ in a humidified atmosphere of $5 \% \mathrm{CO}_{2}$.

\subsection{Preparation of Arc}

Arc was purchased from the National Institute for the Control of Pharmaceutical and Biological Products (Beijing, China; molecular formula: $\mathrm{C}_{21} \mathrm{H}_{24} \mathrm{O}_{6}$; molecular weight: 372.4117) and kept from direct exposure to light and air during the experiments. Stock solution of Arc $(1 \mathrm{mM})$ was dissolved in Phosphate Buffered Saline (PBS) and stored at $-20^{\circ} \mathrm{C}$.

\subsection{Cell Viability}

Human SH-SY5Y cells were pretreated with $\mathrm{H} 89$ for $1 \mathrm{~h}$, followed by exposure to Arc $(0.5 \mu \mathrm{M})$ for $24 \mathrm{~h}$. Cell viability was measured by MTT assay. Briefly, cells were incubated with $0.5 \mathrm{mg} / \mathrm{mL}$ MTT (Sigma, St. Louis, MO, USA) at room temperature (RT) for $4 \mathrm{~h}$. Formed formazan crystals were dissolved in Dimethyl sulfoxide (DMSO), and the plates were analyzed using a microplate reader (MR-96A, Mindray, Shenzhen, China) at $540 \mathrm{~nm}$.

\subsection{Immunofluorescence, Hoechst33258 Staining and Neuritogenesis}

Cells were fixed for 30 min with 4\% paraformaldehyde and washed three times with PBS. The cells were incubated with primary antibody at $4{ }^{\circ} \mathrm{C}$ overnight. The primary antibodies used were as follows: mouse anti-NF-M (1:150; StemCell Technologies, Vancouver, BC, Canada), rabbit anti-SYN1 (1:100), rabbit anti-beta-amyloid (35-42) (1:100) and rabbit anti-p-CREB (1:100; all from Bioss. Inc.; Beijing, China). After rinsing with PBS, cells were incubated with appropriate Cy3, fluorescein isothiocyanate (FITC)-conjugated species-specific secondary antibodies (1:200; all from Jackson ImmunoResearch Lab, West Grove, PA, USA) at RT for $1 \mathrm{~h}$. The cells were mounted with mounting medium (Vector Laboratories, Burlingame, CA, USA) containing DAPI or Hoechst $33258(0.5 \mu \mathrm{g} / \mathrm{mL}$; Sigma, St. Louis, MO, USA). Fluorescence was recorded on an inverted fluorescence microscope (NOVEL NIB-100F, NOVEL, Beijing, China). Fluorescence intensities of SYN, A $\beta$ and p-CREB were measured using Image J software (NIH Image J $1.38 \times$, National Institutes of Health, Bethesda, MD, USA) [31]. Cell apoptosis was assessed by nuclear morphology. Cells with condensed chromatin of fragmented nuclei were counted as apoptotic cells. Cells were counted in triplicate cultures in ten randomly chosen areas under the microscope in 2-4 experiments. The cell counter of the Image $\mathrm{J}$ software (NIH Image J 1.38×, National Institutes of Health, Bethesda, MD, USA) was used to count cells, and mean numbers were used for analysis. The frequencies of apoptotic cells were expressed as the relative percentage per 100 cells [32]. For analysis of neuritogenesis, images were captured over randomly selected fields by using a $40 \times$ objective and constant camera settings within each experiment. At least 10 fields were analyzed for each sample. In each selected field, the numbers of cell bodies and neurites were counted [33]. 


\subsection{Quantitative PCR with Reverse Transcription}

For mRNA quantification, the total RNA of neurons was isolated using TRIzol reagent (Carlsbad, CA, USA). A RevertAid First Strand cDNA Synthesis Kit (Fermentas, Lafayette, CO, USA) was used to synthesize the cDNA. PCR was performed using the DreamTaq Green PCR Master Mix Kit (all from Thermo Scientific, Lafayette, CO, USA). The primers used to detect the expression of $C R E B$ were: 5'-ATA AAG CCT GCA ACA GCC AAC T-3' (forward) and 5'-CAA AGA CCT GCT AAT CCT CAC G-3' (reverse); BACE1 were: 5'-TAG GAT CCA TGG CCC CAG CGC TGC ACT-3' (forward) and 5'-CGG AAT TCT TAC TTG ATC TAA-3'(reverse); PS1 were: 5'-CAA CCC TGA GCC AAT TCA CAA GA-3' (forward) and 5'-CGG GTA TAG AAG CTG ACT GAT-3'(reverse); $\beta$-actin were: 5'-TGC TGT CCC TGT ATG CCT CT-3' (forward) and 5'-TTT GAT GTC ACG CAC GAT TT-3' (reverse). The PCR cycling program parameters were as follows: $30 \mathrm{~s}, 94{ }^{\circ} \mathrm{C} ; 35$ cycles of $30 \mathrm{~s}, 56{ }^{\circ} \mathrm{C} ; 1 \mathrm{~min}, 72{ }^{\circ} \mathrm{C}$. The final extension was separated by electrophoresis on $1.0 \%$ agarose gels. The values obtained for the target gene expression were normalized to $\beta$-actin and quantified relative to the expression in control samples.

\subsection{Quantification of BACE1 and PS1 by ELISA}

$\beta$-secretase (BACE1) and presenilin 1 (PS1) levels were quantified in cell supernatants by an ELISA kit (R \& D) following the manufacturer's instruction.

\subsection{Statistical Analysis}

Results were expressed as the mean \pm SD from an appropriate number of experiments. Statistical evaluation was performed by one-way ANOVA with Bonferroni's multiple comparison test.

\section{Conclusions}

This collective evidence indicates that compared to H89-treated mouse cortical neurons and human SH-SY5Y cells, cells treated with Arc plus H89 showed enhanced cell viability, decreased A $\beta$ production, reduced PS1 protein levels, attenuated cell apoptosis, increased neurite outgrowth and expression of the synaptic marker, SYN, and the underlying mechanism may be attributed to the enhancement of CREB activation.

\section{Acknowledgments}

This work was supported by the grants from the funding of the National Eleventh-Five-Year Plan in R \& D of Important New Medicine (2009ZX09103-423), the National Natural Science Foundation of China (81173580), the Natural Science Foundation of Liaoning Province (201102144), the Project of Talents in Colleges and Universities in Liaoning Province, and the Science Foundation of Shenyang City (F11-264-1-42).

\section{Conflicts of Interest}

The authors declare no conflict of interest. 


\section{References}

1. Lee, J.Y.; Cho, B.J.; Park, T.W.; Park, B.E.; Kim, S.J.; Sim, S.S.; Kim, C.J. Dibenzylbutyrolactone lignans from Forsythia koreana fruits attenuate lipopolysaccharide-induced inducible nitric oxide synthetase and cyclooxygenase-2 expressions through activation of nuclear factor-kappab and mitogen-activated protein kinase in RAW264.7 cells. Biol. Pharm. Bull. 2010, 33, 1847-1853.

2. Tsai, W.J.; Chang, C.T.; Wang, G.J.; Lee, T.H.; Chang, S.F.; Lu, S.C.; Kuo, Y.C. Arctigenin from Arctium lappa inhibits interleukin-2 and interferon gene expression in primary human $\mathrm{T}$ lymphocytes. Chin. Med. 2011, 6, 12.

3. Gu, Y.; Qi, C.; Sun, X.; Ma, X.; Zhang, H.; Hu, L.; Yuan, J.; Yu, Q. Arctigenin preferentially induces tumor cell death under glucose deprivation by inhibiting cellular energy metabolism. Biochem. Pharmacol. 2012, 84, 468-476.

4. Jang, Y.P.; Kim, S.R.; Choi, Y.H.; Kim, J.; Kim, S.G.; Markelonis, G.J.; Oh, T.H.; Kim, Y.C. Arctigenin protects cultured cortical neurons from glutamate-induced neurodegeneration by binding to kainate receptor. J. Neurosci. Res. 2002, 68, 233-240.

5. Lee, I.A.; Joh, E.H.; Kim, D.H. Arctigenin isolated from the seeds of Arctium lappa ameliorates memory deficits in mice. Planta Med. 2011, 77, 1525-1527.

6. Jawhar, S.; Wirths, O.; Bayer, T.A. Pyroglutamate amyloid-beta (Abeta): A hatchet man in Alzheimer disease. J. Biol. Chem. 2011, 286, 38825-38832.

7. Shaywitz, A.J.; Greenberg, M.E. CREB: A stimulus-induced transcription factor activated by a diverse array of extracellular signals. Annu. Rev. Biochem. 1999, 68, 821-861.

8. Silva, A.J.; Kogan, J.H.; Frankland, P.W.; Kida, S. CREB and memory. Annu. Rev. Neurosci. 1998, 21, 127-148.

9. Lonze, B.E.; Ginty, D.D. Function and regulation of CREB family transcription factors in the nervous system. Neuron 2002, 35, 605-623.

10. Pugazhenthi, S.; Wang, M.; Pham, S.; Sze, C.I.; Eckman, C.B. Downregulation of CREB expression in Alzheimer's brain and in Abeta-treated rat hippocampal neurons. Mol. Neurodegener. 2011, 6, 60.

11. Dineley, K.T.; Westerman, M.; Bui, D.; Bell, K.; Ashe, K.H.; Sweatt, J.D. Beta-amyloid activates the mitogen-activated protein kinase cascade via hippocampal alpha7 nicotinic acetylcholine receptors: In vitro and in vivo mechanisms related to Alzheimer's disease. J. Neurosci. 2001, 21, 4125-4133.

12. Funaki, C.; Hodges, R.R.; Dartt, D.A. Identification of the Raf-1 signaling pathway used by cAMP to inhibit p42/p44 MAPK in rat lacrimal gland acini: Role in potentiation of protein secretion. Invest. Ophthalmol. Visual Sci. 2010, 51, 6321-6328.

13. Lai, H.C.; Wu, M.J.; Chen, P.Y.; Sheu, T.T.; Chiu, S.P.; Lin, M.H.; Ho, C.T.; Yen, J.H. Neurotrophic effect of citrus 5-hydroxy-3,6,7,8,3',4'-hexamethoxyflavone: Promotion of neurite outgrowth via cAMP/PKA/CREB pathway in PC12 cells. PLoS One 2011, 6, e28280.

14. Chu, J.; Pratico, D. 5-lipoxygenase as an endogenous modulator of amyloid beta formation in vivo. Annu. Neurol. 2011, 69, 34-46. 
15. Cho, I.J.; Woo, N.R.; Shin, I.C.; Kim, S.G. H89, an inhibitor of PKA and MSK, inhibits cyclic-AMP response element binding protein-mediated MAPK phosphatase-1 induction by lipopolysaccharide. Inflamm. Res. 2009, 58, 863-872.

16. Costes, S.; Broca, C.; Bertrand, G.; Lajoix, A.D.; Bataille, D.; Bockaert, J.; Dalle, S. ERK1/2 control phosphorylation and protein level of cAMP-responsive element-binding protein: A key role in glucose-mediated pancreatic beta-cell survival. Diabetes 2006, 55, 2220-2230.

17. Markou, T.; Hadzopoulou-Cladaras, M.; Lazou, A. Phenylephrine induces activation of CREB in adult rat cardiac myocytes through MSK1 and PKA signaling pathways. J. Mol. Cell. Cardiol. 2004, 37, 1001-1011.

18. Wang, M.D.; Huang, Y.; Zhang, G.P.; Mao, L.; Xia, Y.P.; Mei, Y.W.; Hu, B. Exendin-4 improved rat cortical neuron survival under oxygen/glucose deprivation through PKA pathway. Neuroscience 2012, 226, 388-396.

19. Farrow, B.; Rychahou, P.; Murillo, C.; O’Connor, K.L.; Iwamura, T.; Evers B.M. Inhibition of pancreatic cancer cell growth and induction of apoptosis with novel therapies directed against protein kinase A. Surgery 2003, 134, 197-205.

20. Hou, Y.; Aboukhatwa, M.A.; Lei, D.L.; Manaye, K.; Khan, I.; Luo, Y. Anti-depressant natural flavonols modulate BDNF and beta amyloid in neurons and hippocampus of double TgAD mice. Neuropharmacology 2010, 58, 911-920.

21. Li, S.; Reinprecht, I.; Fahnestock, M.; Racine, R.J. Activity-dependent changes in synaptophysin immunoreactivity in hippocampus, piriform cortex, and entorhinal cortex of the rat. Neuroscience 2002, 115, 1221-1229.

22. Butterfield, D.A. Amyloid beta-peptide (1-42)-induced oxidative stress and neurotoxicity: implications for neurodegeneration in Alzheimer's disease brain. A review. Free Radical Res. 2002, 36, 1307-1313.

23. Zhu, X.; Chen, C.; Ye, D.; Guan, D.; Ye, L.; Jin, J.; Zhao, H.; Chen, Y.; Wang, Z.; Wang, X.; et al. Diammonium glycyrrhizinate upregulates PGC-1alpha and protects against Abeta1-42-induced neurotoxicity. PLoS One 2012, 7, e35823.

24. Vetrivel, K.S.; Thinakaran, G. Amyloidogenic processing of beta-amyloid precursor protein in intracellular compartments. Neurology 2006, 66, S69-S73.

25. Heredia, L.; Helguera, P.; de Olmos, S.; Kedikian, G.; Sola Vigo, F.; LaFerla, F.; Staufenbiel, M.; de Olmos, J.; Busciglio, J.; Caceres, A.; et al. Phosphorylation of actin-depolymerizing factor/cofilin by LIM-kinase mediates amyloid beta-induced degeneration: A potential mechanism of neuronal dystrophy in Alzheimer's disease. J. Neurosci. 2006, 26, 6533-6542.

26. Kudo, T.A.; Kanetaka, H.; Mizuno, K.; Ryu, Y.; Miyamoto, Y.; Nunome, S.; Zhang, Y.; Kano, M.; Shimizu, Y.; Hayashi, H. Dorsomorphin stimulates neurite outgrowth in PC12 cells via activation of a protein kinase A-dependent MEK-ERK1/2 signaling pathway. Genes Cells 2011, $16,1121-1132$.

27. Zheng, Z.; Keifer, J. PKA has a critical role in synaptic delivery of GluR1- and GluR4-containing AMPARs during initial stages of acquisition of in vitro classical conditioning. J. Neurophysiol. 2009, 101, 2539-2549.

28. Delghandi, M.P.; Johannessen, M.; Moens, U. The cAMP signalling pathway activates CREB through PKA, p38 and MSK1 in NIH 3 T3 cells. Cell. Signal. 2005, 17, 1343-1351. 
29. Tan, Y.W.; Zhang, S.J.; Hoffmann, T.; Bading, H. Increasing levels of wild-type CREB up-regulates several activity-regulated inhibitor of death (AID) genes and promotes neuronal survival. BMC Neurosci. 2012, 13, 48.

30. Nie, B.M.; Jiang, X.Y.; Cai, J.X.; Fu, S.L.; Yang, L.M.; Lin, L.; Hang, Q.; Lu, P.L.; $\mathrm{Lu}, \mathrm{Y}$. Panaxydol and panaxynol protect cultured cortical neurons against Abeta25-35-induced toxicity. Neuropharmacology 2008, 54, 845-853.

31. Kwon, M.; Fernandez, J.R.; Zegarek, G.F.; Lo, S.B.; Firestein, B.L. BDNF-promoted increases in proximal dendrites occur via CREB-dependent transcriptional regulation of cypin. J. Neurosci. 2011, 31, 9735-9745.

32. Saeki, K.; Nose, Y.; Hirao, N.; Takasawa, R.; Tanuma, S. Amyloid precursor protein binding protein Fe65 is cleaved by caspases during DNA damage-induced apoptosis. Biol. Pharm. Bull. 2011, 34, 290-294.

33. Dedoni, S.; Olianas, M.C.; Ingianni, A.; Onali, P. Type I interferons impair BDNF-induced cell signaling and neurotrophic activity in differentiated human SH-SY5Y neuroblastoma cells and mouse primary cortical neurons. J. Neurochem. 2012, 122, 58-71.

(C) 2013 by the authors; licensee MDPI, Basel, Switzerland. This article is an open access article distributed under the terms and conditions of the Creative Commons Attribution license (http://creativecommons.org/licenses/by/3.0/). 